Pushpak Chandrakant Chirmade, Sonia Parikh, Asha Anand, Harsha Panchal, Apurva Patel, Sandip Shah

Gujarat Cancer and Research Institute, Ahmedabad, India

\title{
Primary pleuropulmonary synovial sarcoma with brain metastases in a paediatric patient: an unusual presentation
}

The authors declare no financial disclosure

\begin{abstract}
Primary lung neoplasms are rare in children. The most common primary lung malignancies in children are pleuropulmonary blastoma and carcinoid tumour. Synovial sarcoma (SS) accounts for approximately $1 \%$ of all childhood malignancies. In absolute terms, the SS of the lungs and pleura are extremely rare and pose a diagnostic difficulty. Soft tissue sarcomas usually have a high potential for metastases, however, metastasis to the brain is rare, even in widely disseminated disease, and it has been described only in 3 case reports previously. Primary pleuropulmonary SS with brain metastases is even rarer. Here we present a case of an 11-year-old boy who presented with respiratory complaints, viz. fever and cough for 20 days. Initial impression was lung abscess, however, on histopathological, immunohistochemical and molecular study, the disorder was diagnosed as synovial sarcoma. After a week from the first consult, the child developed neurological symptoms, viz., an episode of convulsion and gradually worsening power of the lower limb. Computed tomography scan and Magnetic Resonance Spectroscopy was suggestive of brain metastases. Given the rarity of primary lung neoplasms in children, clinical detection remains a challenge. Delayed diagnoses are common as respiratory symptoms may be attributed to inflammatory or infective processes. Primary pleuropulmonary synovial sarcoma is a rare tumour and it is not known to commonly metastasise to the brain. Though rare, primary pleuropulmonary SS should be considered an important differential among peadiatric primary lung neoplasms due to its potential for curability if detected early, and more aggressive metastatic pattern, e.g. brain metastases making early detection imperative.
\end{abstract}

Key words: peadiatric sarcoma, primary pleuropulmonary synovial sarcoma, brain metastases, SS18 rearrangement, TLE positive

Adv. Respir. Med. 2017; 85: 206-210

\section{Introduction}

Primary pleuropulmonary synovial sarcoma is an extremely rare tumour. Most of the primary lung malignancies are carcinomas. Sarcomas comprise only $0.5 \%$ of primary lung malignancies $[1,2]$. Soft tissue sarcomas have a very high tendency to metastasise, the most common site being the lung. The brain is a rare location of metastases. Here we present a rare case of a child with primary pleuropulmonary synovial sarcoma with brain metastases.

\section{Case report}

An 11-year-old boy presented with the chief complaints of moderate grade intermittent fever, without chills and cough with scanty, non purulent, watery expectoration for approximately 20 days. Investigation by a local paediatrician, chest X-ray (CXR) and ultrasonography (USG) of the thorax showed right lower zone consolidation with moderate pleural effusion. An intercostal drain was inserted and patient was referred to a multidisciplinary centre. 

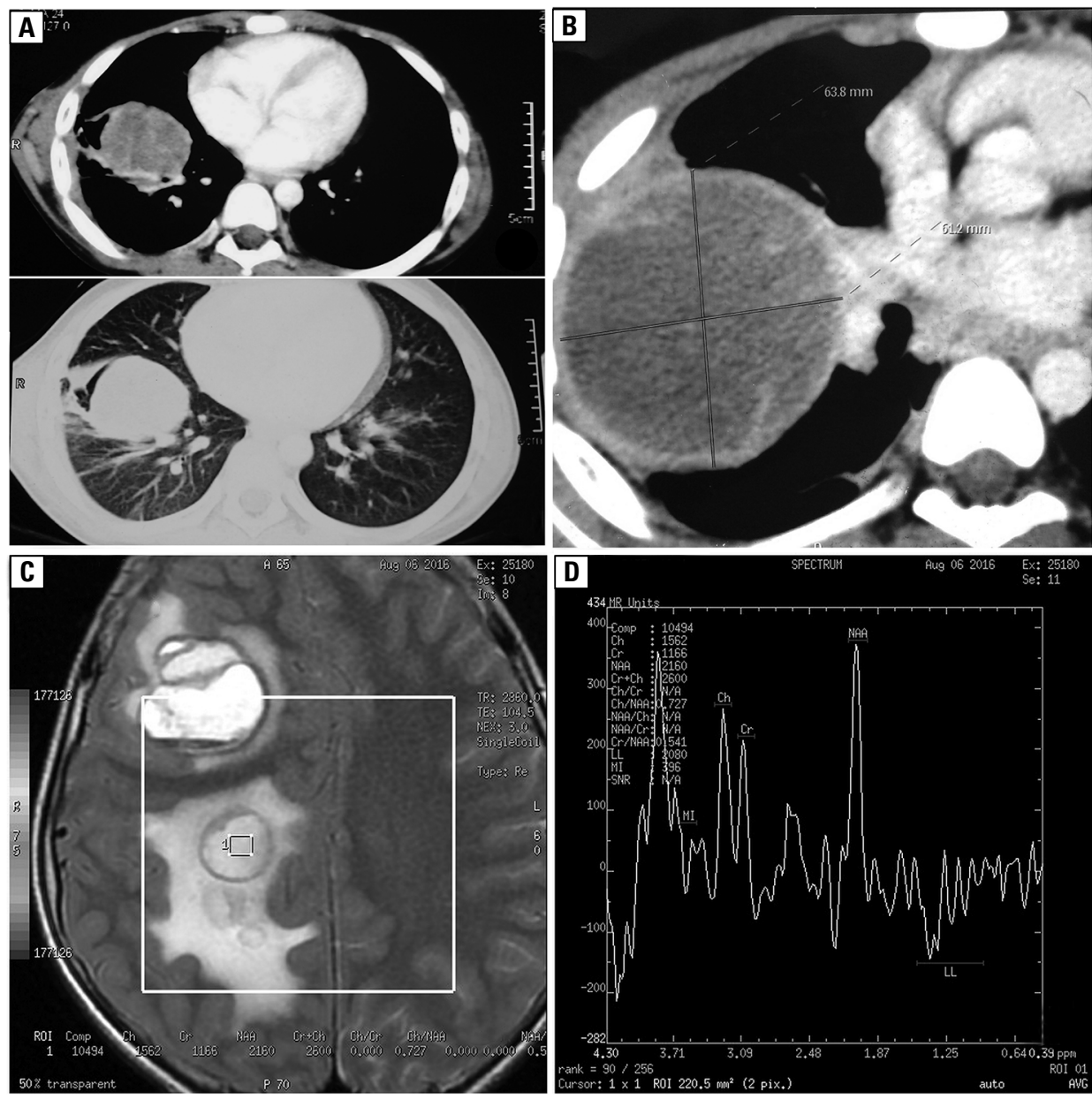

Figure 1. A, B - CT scan of thorax showing pulmonary mass; C, D - MR spectroscopy findings

According to records, at presentation, the child was normotensive but sick-looking with tachypnea and tachycardia. Saturation of haemoglobin with oxygen was $96 \%$. The patient was started on intravenous (iv) antibiotics and iv fluids. A complete haemogram, liver and renal function tests, and the results of blood cultures were within normal limits.

A computed tomography (CT) scan of the thorax was done, which showed a large well-defined multiloculated thin walled peripherally enhancing necrotic lesion $(63 \mathrm{~mm} \times 62 \mathrm{~mm} \times$ $76 \mathrm{~mm}$ ) in the right middle and lower lobe with few air foci in between, suggesting the possibility of lung abscess or cystic metastases (Fig. 1A, B).

At the multidisciplinary centre; considering it lung abscess, a thoracoscopy with right posterolateral thoracotomy was performed. Necrotic material from the right upper and middle lobe was drained, decortication of the pleura was done and a 24 French intercostal drain was kept in situ. The material was sent for routine exam, microbiological testing, culture and histopathological evaluation
(HPE). HPE was suggestive of malignancy so patient was referred to our regional cancer centre.

The histopathological diagnosis review was indicative of high grade sarcoma with spindle and epithelioid morphology, thus suggestive of biphasic synovial sarcoma (SS). Immunohistochemistry (IHC) panel showed CD99, TLE-1, EMA, CK positivity while SAL4, SOX10, CD56, OCT3/4, desmin and myogenin were negative (Fig. $2 \mathrm{~A}-\mathrm{D}$ ). In the absence of cartilage in specimen and relatively older age of the patient, the differential of pleuropulmonary blastoma was ruled out.

A thorough search for primary disease of the extremities was done. Surgery was attempted to resect the primary lesion, but the lesion was extensive and involved the pleura and subcutaneous tissue. Thus only biopsy was taken which revealed high-grade synovial sarcoma. A repeat IHC panel showed vimentin, CD99, Bcl 2 positivity, whereas desmin, actin, S-100, AE-1, synaptophysin were negative. The FFPE block was sent for FISH SS18 rearrangement, which was positive, hence confirming diagnosis of synovial sarcoma. 


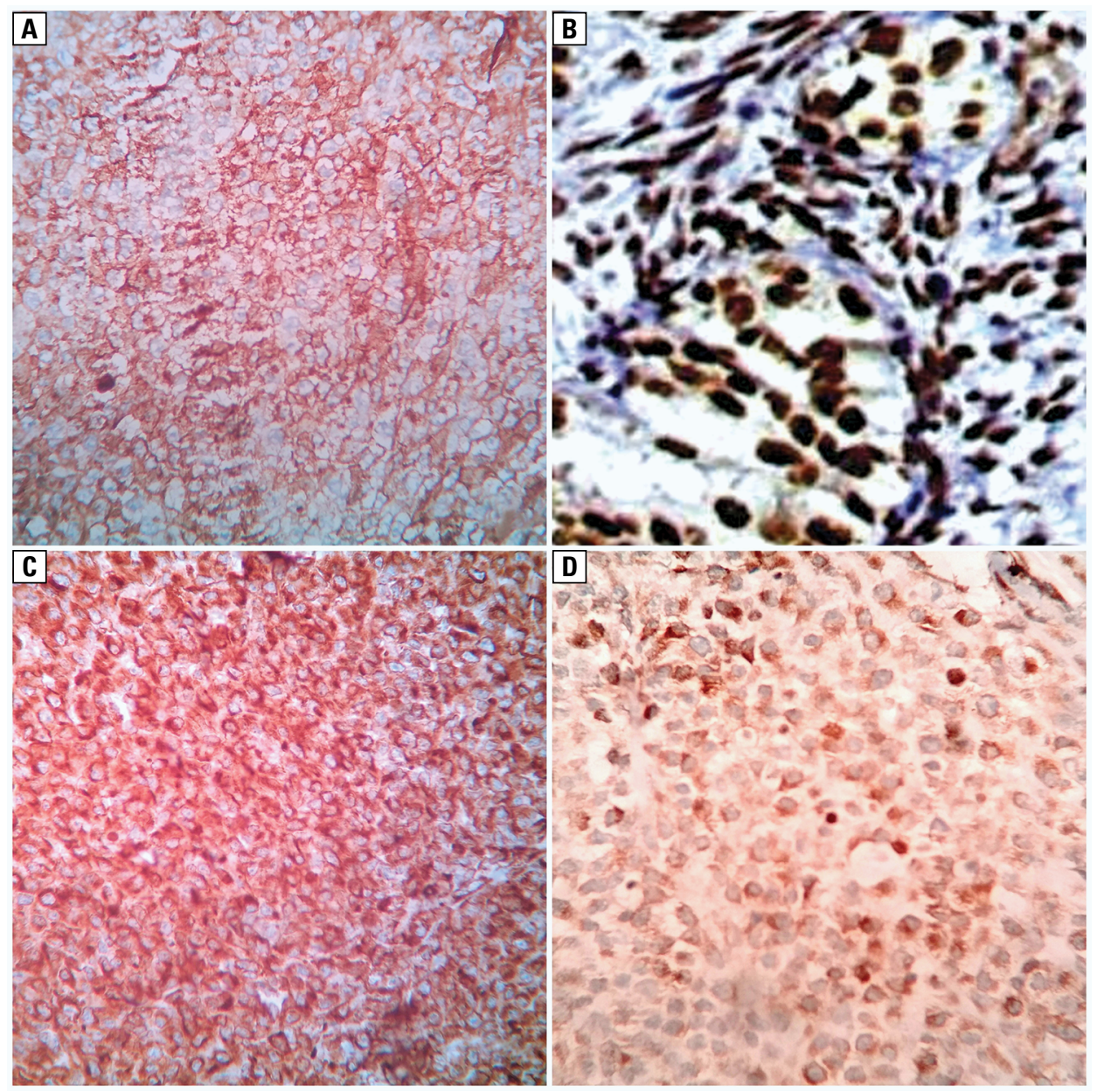

Figure 2. A — CD99 membranous positivity; B — TLE nuclear strong positivity; C — Vimentin cytoplasmic plus membranous positivity; D — BCI2 nuclear positivity

After a week from the first consult, the child presented with an episode of convulsion and gradually worsening power in the left lower limb, which worsened from grade 4 to grade 2 within a span of 2 days. The child was started on anti cerebral oedema measures (injectable dexamethasone and mannitol) and anti-convulsant drugs. A contrast enhanced CT scan of the brain showed multiple peripherally enhancing hypodense ring enhancing lesions in the right frontal and parietal lobes, the largest measuring $31 \mathrm{~mm} \times 41 \mathrm{~mm}$ with cerebral oedema and midline shift of $10 \mathrm{~mm}$.

In view of frugal literature suggesting synovial sarcoma metastasising to the brain, alternate diagnoses, viz., tuberculoma, neurocysticercosis were considered. Magnetic resonance imaging with spectroscopy (MRS) of the brain was ordered, which showed elevated Cholin (Cho) level with mildly reduced $\mathrm{N}$ - acetylaspartate (NAA) level with the increase in the Cho/NAA and Cho/total creatine (Cr) ratio suggestive of neoplastic aetiology (Fig. 2C, D).

In view of circumstantial evidence, a diagnosis of SS with brain metastases was made.
Neurosurgery opinion was taken for assessment of resectability of brain metastases; however, complete resection of all intracranial lesions was not possible.

Biopsy of the intracranial lesion was not contemplated as enough circumstantial evidence was gathered in the form of MRS findings which suggested neoplastic etiology; other factors taken into account were unstable neurological status of the patient, aggressive nature of tumour and morbidity of the procedure.

The patient was referred for whole brain irradiation. Radiotherapy with a total dose of 30 Grays over 10 fractions was administered. The child recovered neurologically with grade 4 plus power, able to walk without support. Subsequently, he has been started on systemic chemotherapy with palliative intent comprising of single agent doxorubicin $\left(25 \mathrm{mg} / \mathrm{m}^{2}\right)$ for 3 days. The patient tolerated the first two cycles of chemotherapy very well, thus, ifosfamide $\left(3 \mathrm{~g} / \mathrm{m}^{2}\right)$ for 3 days was added to doxorubicin. After total 4 cycles of chemotherapy, re-evaluation showed stable 
pulmonary disease and partial response in brain metastases according to RECIST 1.1 guidelines. The patient has been ambulatory without support with minimal pulmonary symptoms, however, he had one episode of breakthrough convulsion.

\section{Discussion}

Primary lung neoplasms are rare in children. The common types of lung cancer in adults are exceptional occurrences in the paediatric population. The ratio of primary tumours to metastatic to non-neoplastic lesions in pathologic changes arising from the lung in children is 1:5:60 [3]. The most common benign pulmonary tumour of the paediatric lung is inflammatory myofibroblastic tumour (52\%), and the most common primary lung malignancies in children are pleuropulmonary blastoma and carcinoid tumour [4].

Synovial sarcoma (SS) accounts for approximately $5-10 \%$ of all soft tissue sarcomas [5]. The incidence of SS is 2.75 per 100,000 individuals [6]. It is the disease of adolescents and young adults, the mean age of diagnoses being 35.4 years [7]. Most common sites of synovial sarcoma are the extremities especially the large joints; however, other locations, viz. head \& neck, lung, mediastinum, and abdominal wall have been reported too. Synovial sarcoma accounts for approximately $1 \%$ of all childhood malignancies and despite its rarity, is the most common non-rhabdomyosarcoma in childhood, making up approximately $30 \%$ of all soft tissue sarcomas [8-10].

In absolute terms, the SS of the lungs and the pleura are extremely rare [6, 7], and pose a diagnostic difficulty. Review of literature shows only few case reports and small case series that report tumours of similar lineage [1, 11-13]. The English literature documents barely 60 cases of synovial sarcoma at pleuropulmonary region till 2005 [14]. Spurrell et al. [15] at Royal Marsden Hospital, London, between 1978 and 2003, presented analysis of 104 cases of SS, out of which only 10 were pleuropulmonary in origin. Pleuropulmonary SS seem to be more aggressive as compared to their counterparts of the extremities with less chance of complete resection and difficulty to achieve adequate wide margins and hence high percentage of local recurrence [16].

Soft tissue sarcomas usually have a high potential to metastasize. The most common site of metastases is the lung. One study showed a 75:1 ratio of lung to liver metastases, as the site of distant spread, however, in this subset with hepatic metastases $22 \%$ had primary abdominal
SS [17]. Similarly, metastasis to the brain is also rare, even in widely disseminated disease [18, 19], and it was reported only in 3 case reports previously [20]. Pleuropulmonary SS with brain metastases is even rarer with just one report mentioning brain metastases as a terminal event [21] and one by Nuwal et al. [22] reported in 2012. The both patients reported were adults and this is probably the first case, to the best of our knowledge, in peadiatric age group and based on pubmed database search to date.

Histopathological variants of SS are biphasic, monophasic epithelial, monophasic fibrous, poorly differentiated. The two main variants are the monophasic and the biphasic types. Biphasic pattern of synovial sarcoma consists of an admixture of glandular structures lined by cuboidal or columnar epithelium set in a sarcomatous stroma [23].

Synovial sarcomas show a specific chromosomal translocation, $\mathrm{t}(\mathrm{X}: 18)(\mathrm{p} 11.2 ; \mathrm{q} 11.2)$, which results in fusion of the SYT gene on the long arm of chromosome 18 with the SSX1, SSX2 or SSX4 gene on the short arm of the X chromosome. This is often helpful to make a diagnosis when SS arises from an unusual site, e.g. the pleura or the lung. Biphasic variants expresses, in most cases, SYTSSX1 gene fusion, while the monophasic type may express both (SYT-SSX1, SYT-SSX2) [24].

Synovial sarcoma demonstrates nuclear staining with TLE1 (transducin-like enhancer of split-1) in $95 \%$ of tumours and appears to be a very sensitive marker. TLE1 encodes for a transcriptional co-repressor that is involved in epithelial and neuronal differentiation. In synovial sarcoma there is usually co-expression of mesenchymal (vimentin) and epithelial markers (cytokeratin and EMA). Only synovial sarcoma is positive for cytokeratin 7 and 19, other soft tissue sarcomas show positivity for cytokeratin 8 and 18; In monophasic synovial sarcoma immunoreactivity of cytokeratin is reduced by almost $60 \%$; Cytokeratin positivity is noted in only $50 \%$ cases of PD synovial sarcoma while EMA positivity in $72 \%$ (more sensitive than cytokeratin), S100 protein positivity in some cases, CD99 positivity in 2/3rd of cases and Bcl-2 positivity in most cases. [23-25]. The immunohistochemistry (IHC) panel in the above mentioned case showed CD99, TLE1, EMA, CK, vimentin, Bcl2 positivity. S100, however, was negative.

The standard care of synovial sarcoma includes surgical resection with adequate free margins to prevent local recurrence followed with adjuvant chemotherapy with or without radiation. 
In our patient, surgical resection of lung primary tumour could not be performed due to extensive involvement of adjacent structures. In view of neurosurgical resection being not possible, the boy received whole brain radiotherapy for brain metastases and was subsequently started on palliative chemotherapy with single agent doxorubicin $\left(25 \mathrm{mg} / \mathrm{m}^{2}\right.$ for 3 days). After 2 cycles of chemotherapy, in view of good tolerance, ifosfamide ( $3 \mathrm{~g} / \mathrm{m}^{2}$ for 3 days) was added to doxorubicin along with appropriate supportive care as per the standard guidelines.

\section{Conclusions}

Given the rarity of primary lung neoplasms in children, clinical detection remains a challenge. Delayed diagnoses are common as respiratory symptoms may be attributed to inflammatory or infective processes. Primary pleuropulmonary synovial sarcoma, though rare, should be considered as an important differential among peadiatric primary lung neoplasms for its potential for curability if detected early. Also primary pleuropulmonary sarcoma is more aggressive and has different metastatic pattern, e.g. brain metastases as in the above mentioned case; compared to synovial sarcoma arising from the extremities making early diagnosis imperative.

\section{Conflict of interest}

The authors declare no conflict of interest.

\section{References:}

1. Keel SB, Bacha E, Mark EJ, et al. Primary pulmonary sarcoma: a clinicopathologic study of 26 cases. Mod Pathol. 1999; 12(12): 1124-1131, indexed in Pubmed: 10619264.

2. Etienne-Mastroianni B, Falchero L, Chalabreysse L, et al. Primary sarcomas of the lung: a clinicopathologic study of 12 cases. Lung Cancer. 2002; 38(3): 283-289, indexed in Pubmed: 12445750.

3. Cohen MC, Kaschula RO. Primary pulmonary tumors in childhood: a review of 31 years' experience and the literature. Pediatr Pulmonol. 1992; 14(4): 222-232, indexed in Pubmed: 1336597.

4. Hancock BJ, Di Lorenzo M, Youssef S, et al. Childhood primary pulmonary neoplasms. J Pediatr Surg. 1993; 28(9): 1133-1136, indexed in Pubmed: 8308677.

5. Eilber FC, Dry SM. Diagnosis and management of synovial sarcoma. J Surg Oncol. 2008; 97(4): 314-320, doi: 10.1002/ jso.20974, indexed in Pubmed: 18286474.

6. Sakellaridis N, Mahera H, Pomonis S. Hemangiopericytoma-like synovial sarcoma of the lumbar spine. Case report. J Neurosurg Spine. 2006; 4(2): 179-182, doi: 10.3171/spi.2006.4.2.179, indexed in Pubmed: 16506487.

7. Krieg AH, Hefti F, Speth BM, et al. Synovial sarcomas usually metastasize after $>5$ years: a multicenter retrospective analysis with minimum follow-up of 10 years for survivors. Ann Oncol. 2011; 22(2): 458-467, doi: 10.1093/annonc/mdq394, indexed in Pubmed: 20716627.
8. Greene S, Hawkins DS, Rutledge JC, et al. Pediatric intradural extramedullary synovial sarcoma: case report. Neurosurgery. 2006; 59(6): E1339; discussion E1339, doi: 10.1227/01. NEU.0000245619.24603.96, indexed in Pubmed: 17277671.

9. McGrory JE, Pritchard DJ, Arndt CA, et al. Nonrhabdomyosarcoma soft tissue sarcomas in children. The Mayo Clinic experience. Clin Orthop Relat Res. 2000(374): 247-258, indexed in Pubmed: 10818984.

10. Koss M, Travis W, Moran C. Pulmonary sarcomas, blastoma, carcinosarcomas and teratomas. In: Hasleton PS, Travis W, Moran C. ed. Spencer's pathology of the lung. 5th ed. McGraw Hill, New York 1996: 1065-1069.

11. Collins BT, Janney CG, Ong M, et al. Fine needle aspiration biopsy of monophasic spindle synovial sarcoma of lung with fluorescence in situ hybridization identification of $t(x ; 18)$ translocation: a case report. Acta Cytol. 2009; 53(1): 105-108, indexed in Pubmed: 19248564.

12. Zamarrón C, Abdulkader I, Alvarez UC, et al. Primary synovial sarcoma of the lung. Intern Med. 2006; 45(10): 679-683, indexed in Pubmed: 16778340.

13. Zeren H, Moran CA, Suster S, et al. Primary pulmonary sarcomas with features of monophasic synovial sarcoma: a clinicopathological, immunohistochemical, and ultrastructural study of 25 cases. Hum Pathol. 1995; 26(5): 474-480, indexed in Pubmed: 7750931.

14. Hosono T, Hironaka M, Kobayashi A, et al. Primary pulmonary synovial sarcoma confirmed by molecular detection of SYT-SSX1 fusion gene transcripts: a case report and review of the literature. Jpn J Clin Oncol. 2005; 35(5): 274-279, doi: 10.1093/ jico/hyi073, indexed in Pubmed: 15879502.

15. Spurrell EL, Fisher C, Thomas JM, et al. Prognostic factors in advanced synovial sarcoma: an analysis of 104 patients treated at the Royal Marsden Hospital. Ann Oncol. 2005; 16(3): 437-444, doi: 10.1093/annonc/mdi082, indexed in Pubmed: 15653701.

16. Essary L, Vargas S, Fletcher C. Primary pleuropulmonary synovial sarcoma. Cancer. 2002; 94(2): 459-469, doi: 10.1002/cncr.10188.

17. Jaques DP, Coit DG, Casper ES, et al. Hepatic metastases from soft-tissue sarcoma. Ann Surg. 1995; 221(4): 392-397, indexed in Pubmed: $\underline{7726675}$.

18. Bakri A, Shinagare AB, Krajewski KM, et al. Synovial sarcoma: imaging features of common and uncommon primary sites, metastatic patterns, and treatment response. AJR Am J Roentgenol. 2012; 199(2): W208-W215, doi: 10.2214/AJR.11.8039, indexed in Pubmed: 22826423.

19. Kaufman J, Tsukada Y. Synovial sarcoma with brain metastases. Report of a case responding to supervoltage irradiation and review of the literature. Cancer. 1976; 38(1): 96-99, indexed in Pubmed: 181133.

20. Siegel HJ, Dunahm WH, Lopez-Ben R, et al. Intracranial metastasis from synovial sarcoma. Orthopedics. 2008; 31(4): 405-407, indexed in Pubmed: 19292263.

21. Boroumand N, Raja V, Jones DV, et al. SYT-SSX2 variant of primary pulmonary synovial sarcoma with focal expression of CD117 (c-Kit) protein and a poor clinical outcome. Arch Pathol Lab Med. 2003; 127(4): e201-e204, doi: 10.1043/0003-9985(2003)127<e201:SVOPPS>2.0.CO;2, indexed in Pubmed: 12683902.

22. Nuwal P, Dixit R, Shah NS, et al. Primary monophasic synovial sarcoma lung with brain metastasis diagnosed on transthoracic FNAC: Report of a case with literature review. Lung India. 2012; 29(4): 384-387, doi: 10.4103/0970-2113.102841, indexed in Pubmed: 23243358.

23. Weiss SW, Goldblum JR. Enzinger's and Weiss's soft tissue tumors. 5th ed. . Mosby Elsevier, Philadelhia 2008: 1161-1182.

24. Mikami Y, Nakajima M, Hashimoto H, et al. Primary poorly differentiated monophasic synovial sarcoma of the lung. A case report with immunohistochemical and genetic studies. Pathol Res Pract. 2003; 199(12): 827-833, doi: 10.1078/0344-033800502, indexed in Pubmed: 14989495.

25. Hirakawa N, Naka T, Yamamoto I, et al. Overexpression of bcl-2 protein in synovial sarcoma: a comparative study of other soft tissue spindle cell sarcomas and an additional analysis by fluorescence in situ hybridization. Hum Pathol. 1996; 27(10): 1060-1065, indexed in Pubmed: 8892591. 